\title{
Distance Product Cubics
}

\section{Distance Product Cubics ABSTRACT}

The locus of points that determine a constant product of their distances to the sides of a triangle is a cubic curve in the projectively closed Euclidean triangle plane. In this paper, algebraic and geometric properties of these distance product cubics shall be studied. These cubics span a pencil of cubics that contains only one rational and non-degenerate cubic curve which is known as the Bataille acnodal cubic determined by the product of the actual trilinear coordinates of the centroid of the base triangle. Each triangle center defines a distance product cubic. It turns out that only a small number of triangle centers share their distance product cubic with other centers. All distance product cubics share the real points of inflection which lie on the line at infinity. The cubics' dual curves, their Hessians, and especially those distance product cubics that are defined by particular triangle centers shall be studied.

Key words: triangle cubic, elliptic cubic, rational cubic, trilinear distance, constant product, Steiner inellipse, triangle centers

MSC2010: 51M04 51N35 14H52 14N25

\section{Introduction}

Cubics occur frequently in triangle geometry. Sometimes, cubics are defined as locus of points satisfying certain geometric or algebraic conditions. There are many wellknown cubics such as the Neuberg cubic, the Thomson cubic, the Darboux cubic to name just the most prominent examples. These triangle cubics are known to carry some triangle centers together with points related to the triangle, and besides their (in principle) Euclidean generation, some of them allow for a projective generation, cf. [8]. In many cases, these cubics pass through the vertices of the base triangle: For example, the Thomson cubic $\mathcal{K}_{002}$ (sometimes called seventeen-point cubic, illustrated in Figure 1) passes

\section{Kubike konstantnog umnoška udaljenosti SAŽETAK}

U projektivno zatvorenoj Euklidskoj ravnini trokuta geometrijsko mjesto točaka trokuta kojima je umnožak udaljenosti od stranica trokuta konstantan je jedna kubika. Proučavat će se algebarska i geometrijska svojstva tih kubika konstantnog umnoška udaljenosti. Takve kubike čine pramen kubika koje sadrže samo jednu racionalnu nedegeneriranu kubiku poznatu kao Batailleova kubika s izoliranom točkom, a koja je određena umnoškom pravih trilinearnih koordinata težišta temeljnog trokuta. Svaka točka trokuta određuje jednu kubiku konstantnog umnoška udaljenosti. Ispostavlja se da mali broj točaka trokuta međusobno dijele kubiku konstantnog umnoška udaljenosti. Sve kubike konstantnog umnoška udaljenosti dijele realne točke infleksije koje leže na pravcu u beskonačnosti. Proučavat će se dualne krivulje kubike, njihove Hessianove matrice i posebno one kubike konstantnog umnoška udaljenosti koje su određene poznatim točkama trokuta.

Ključne riječi: kubika trokuta, eliptična kubika, racionalna kubika, trilinearna udaljenost, konstantni umnožak, Steinerova upisana elipsa, točke trokuta

through the vertices of the base triangle and carries the triangle centers $X_{i}$ with Kimberling indices

$$
\begin{gathered}
i \in\{1,2,3,4,6,9,57,223,282,1073 \\
1249,3341,3342,3343,3344,3349,3350, \\
3351,3352,3356,14481,39161,39162\},
\end{gathered}
$$

the midpoints of $\Delta$ 's sides, the midpoints of $\Delta$ 's altitudes, the vertices of the Thomson triangle, and the excenters (which are actually 38 points), see [4]. The numbering of triangle centers follows the exhaustive Encyclopedia of Triangle Centers by CLARK KIMBERLING, see [6, 7]. For example, the triangle centers $X_{39161}$ and $X_{39162}$ are the real foci of the inscribed Steiner ellipse $e$. On the other hand, 
the names and numbers of triangle cubics are taken from BERNARD GIBERT's pages [2].

Further, $\mathcal{K}_{002}$ is a self-isogonal cubic with the centroid $X_{2}$ of $\Delta$ as its pivot point. 26 geometric definitions of the Thomson cubic can be found on GIBERT's page [4], dedicated exclusively to the Thomson cubic.

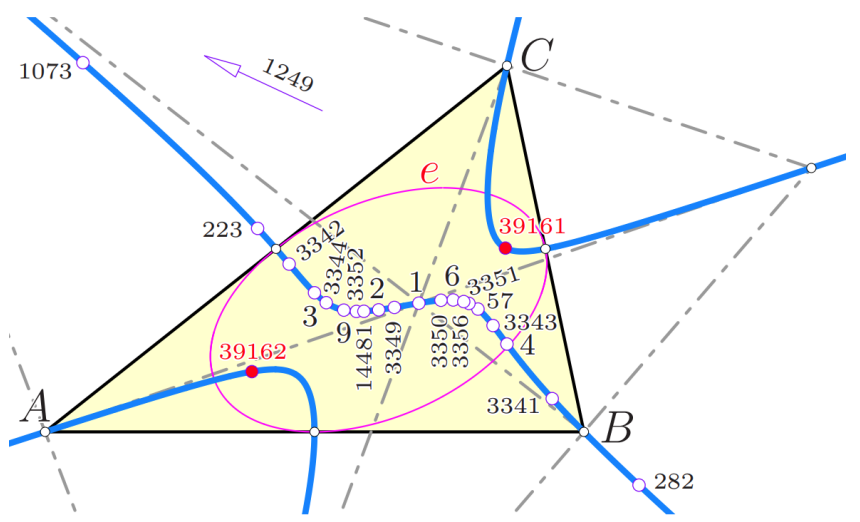

Figure 1: The Thomson cubic $\mathcal{K}_{002}$ with 23 triangle centers on it.

The cubics which shall be studied here, do not pass through the vertices of the triangle. Moreover, the number of triangle centers located on these cubics is rather small except in one case. In many cases, it is impossible to find more than one triangle center on such a cubic. Nevertheless, it is surprising that no one has payed attention to the set of points forming a constant product of distances to the triangle sides.

What is the reason for the interest especially in these curves? It is well-known that elliptic cubics carry a group structure. The operation on the set of points on an elliptic cubic can be seen as an addition. Furthermore, it is well known that these groups contain finitely generated subgroups, cf. [10]. Generators of these groups of finite order are highly sought after. Once, rational (or polynomial) points on elliptic curves are known, many more of them can be generated by simply doubling the initial points. Until now, only a few examples of finitely generated groups on elliptic curves are known. Within the huge amount of triangle cubics carrying rational points, it may be possible to find some more examples.

The paper is organized as follows: In the remaining part of this section, the equation of the distance product cubics are determined. Further, some geometric properties of these particular cubics are deduced. Then, the equations of the dual curves and the curves in the Hessian pencil are given. For the sake of completeness, the Weierstraß normal form of the distance product cubics is derived. Section 2 deals with the very special distance product cubics defined by triangle centers. A complete list (as to November 2020) of groups of triangle centers sharing their distance product cubics is given. It is described how these centers on such cubics can be found in an efficient way and attention is paid to special configurations of triangle centers on their respective distance product cubics. Then, in Section 3 , some (until now) unknown triangle centers on some distance product cubics that contain only one known triangle center are given. Only triangle centers with a relatively short trilinear center function (homogeneous polynomial in the three side lengths $a, b, c$ ) shall be listed. Finally, Section 4 will outline future work and discusses computational problems and challenges. The present paper is an extension and completion of [9].

\subsection{Prerequisites}

In triangle geometry, trilinear coordinates proved useful. For that purpose, the vertices $A, B$, and $C$ of the base triangle $\Delta$ (with side lengths $a=\overline{B C}, b=\overline{C A}, c=\overline{A B}$ ) are described by the homogeneous coordinates

$$
A=(1,0,0), B=(0,1,0), C=(0,0,1),
$$

i.e., the vectors of the canonical basis in $\mathbb{R}^{3}$. The projective frame shall be completed by choosing

$$
X_{1}=(1,1,1)
$$

as the unit point. At this point, it shall be said that the centroid $X_{2}$ of $\Delta$ (like any other center) can also serve as the unit point. With $X_{2}$ as the unit point, barycentric coordinates of points in the plane of the triangle are well-defined, cf. [6]. In the following, trilinear coordinates are preferred, since distances of points to the sides of the base triangle are involved.

Each point $X$ in the plane of $\Delta$ can be uniquely determined by its homogeneous trilinear coordinates

$$
X=(\xi, \eta, \zeta) \neq(0,0,0),
$$

which are the ratios of the oriented distances of $X$ to $\Delta$ 's oriented side lines. The side lines are oriented as $A B$ (from $A$ to $B$ ), $B C$, and $C A$. From homogeneous trilinear coordinates, the (inhomogeneous) actual trilinear coordinates $\left(\xi^{a}, \eta^{a}, \zeta^{a}\right)$ consisting of the three oriented distances of $X$ to $\Delta$ 's side lines can be computed by

$\left(\xi^{a}, \eta^{a}, \zeta^{a}\right)=\frac{2 F}{a \xi+b \eta+c \zeta}(\xi, \eta, \zeta)$,

where $F$ equals the area of the triangle. This normalization fails if

$$
\omega: a \xi+b \eta+c \zeta=0 .
$$

This is the equation of the ideal line $\omega$ (line at infinity) and all points $(\xi, \eta, \zeta)$ on it are ideal points (points at infinity). These points shall be excluded from the following considerations (although there are more than one thousand triangle centers on the ideal line), cf. [7]. 


\subsection{Basic properties}

If one multiplies the actual trilinear coordinates of a point in the plane of the triangle and sets this product equal to a constant $\delta \in \mathbb{R}$, it is possible to state:

Theorem 1 The locus of points $X$ in the (Euclidean) plane of the triangle $\Delta$ that form a constant product $\delta \in \mathbb{R} \backslash\{0\}$ of distances to the side lines of $\Delta$ is a planar cubic curve with the equation

$k_{\delta}: 8 F^{3} \xi \eta \zeta-\delta(a \xi+b \eta+c \zeta)^{3}=0$

in terms trilinear coordinates.

Proof. The equation (2) is obtained by multiplying $\xi^{a}, \eta^{a}$, $\zeta^{a}$ from (1)

$$
\xi^{a} \eta^{a} \zeta^{a}=\frac{8 F^{3}}{(a \xi+b \eta+c \zeta)^{3}}
$$

and, subsequently, setting this product equal to $\delta \in \mathbb{R} \backslash\{0\}$. The equation (2) is homogeneous and of degree three, and thus, it describes a planar cubic curve in the projective plane. A simple computation shows that if (2) is fulfilled by the actual trilinear coordinates of a point, then it is also fulfilled by an arbitrary multiple of these coordinates of the same point, and vice versa.

The equations (2) of the distance product cubics depend linearly on one parameter $\delta \in \mathbb{R} \backslash\{0\}$. Thus, the distance product cubics form a pencil of cubics. Replacing the inhomogeneous parameter $\delta$ in (2) by a homogeneous parameter $\delta=\delta_{1} \delta_{0}^{-1}$ (with $\delta_{0}: \delta_{1} \neq 0: 0$ ), shows that there are two degenerate cubics in the pencil:

(i) If $\delta_{0}: \delta_{1}=1: 0$, the equations of the cubics simplify to

$$
\xi \eta \zeta=0
$$

which is the equation of the union of $\Delta$ 's side lines.

(ii) In the case $\delta_{0}: \delta_{1}=0: 1$, remainder of (2) equals

$$
(a \xi+b \eta+c \zeta)^{3}=0
$$

which is the equation of the ideal line $\omega$ with multiplicity 3 . Figure 2 shows a projective view of a few cubics from the pencil together the three common (collinear) real points of inflection $I_{1}, I_{2}, I_{3}$.

From the fact that the equations of the distance product cubics are linear combinations of $\xi \eta \zeta=0$ and $(a \xi+b \eta+$ $c \zeta)^{3}=0$, it is clear that the ideal points of $\Delta$ 's side lines are the ideal points of the distance product cubics. Furthermore, the intersection of either side line with the each cubic in the pencil is of multiplicity three: For example, $\xi=0$ yields $(b \eta+c \zeta)^{3}=0$, and therefore, $I_{1}=(0,-c, b)$ as the intersection point with multiplicity three.

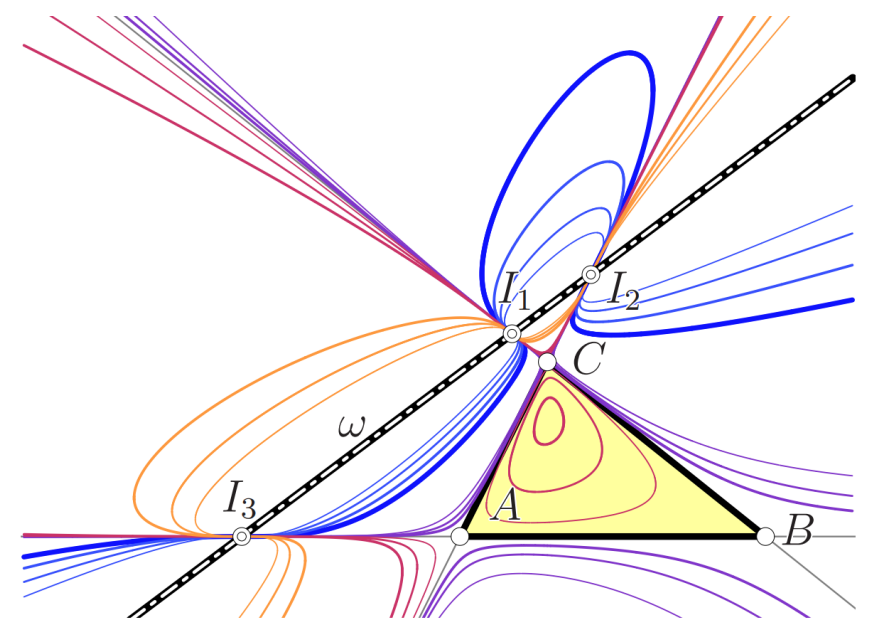

Figure 2: A projective view onto the pencil of distance product cubics shows the three real points of inflection $I_{1}, I_{2}, I_{3}$ on $\omega$.

Theorem 2 The distance product cubics (2) share the three real inflection points, which are at the same time the three ideal points of the cubis. The homogeneous trilinear coordinates of the points of inflection are

$$
I_{1}=(0,-c, b), I_{2}=(c, 0,-a), I_{3}=(-b, a, 0) .
$$

The harmonic polar of a regular point $P \in k$ with respect to a non-degenerate cubic curve $k$ is defined in the following way: Let $P$ be a point on the cubic $k$ and let $l$ be a line through $P$ different from the tangent of $k$ at $P$. Then, in general, $l$ meets the cubic in two further points, say $Q$ and $R$. Provided, that $Q \neq R$ ( $l$ is not tangent to $k$ at some point off $P$ ) and $Q, R \neq P$ ( $l$ is not an inflection tangent), then there exists exactly one point $S$ which is the harmonic conjugate of $P$ with respect to $Q$ and $R$. The locus of $S$ for all $l$ in the pencil about $P$ is called the harmonic polar of $P$ with respect to $k$. The harmonic polars of the inflection points on cubics are straight lines, cf. [1]. In the case of the distance product cubics, the three harmonic polars corresponding to the three real inflection points have a special geometric meaning:

Theorem 3 The harmonic polars of the three inflection points of the distance product cubics are the medians of the base triangle independent of the choice of $\delta$.

Proof. The lines $l$ pencil about $I_{1}=(0,-c, b)$ can be parametrized by

$$
\mathbf{I}(\lambda, \mu)=\lambda(0,-c, b)+\mu(u, v, w)
$$

with $\lambda: \mu \neq 0: 0$, where it means no restriction to assume that $Q=(u, v, w)$ is a further point on $k$ with equation (2). Now, the intersection $R$ of any $l$ with the cubics equals

$$
R=\left(b c u, c^{2} w, b^{2} v\right)
$$


Hence, the harmonic conjugate of $I_{1}$ with respect to $k$ is the point

$$
S=(2 b c u, c(b v+c w), b(b v+c w)) .
$$

The point $S$ lies on the line $b \eta-c \zeta=0$, which is the me$\operatorname{dian}$ through $A$. In the same way it can be shown that the harmonic polars of $I_{2}$ and $I_{3}$ are the medians through $B$ and $C$, respectively. Obviously, the harmonic polar of $I_{1}$ is independent of $\delta$, and so are the harmonic polars of $I_{2}$ and $I_{3}$.

Since the three harmonic polars corresponding to the real inflection points common to all cubics are the medians, the centroid $X_{2}$ must have a special meaning for the distance product cubics. Now, the following can be shown:

Theorem 4 The distance product cubic $k_{2}$ through the centroid of $\Delta$ is the only rational cubic (among the regular ones) in the pencil and the centroid is an isolated node on $k_{2}$.

Remark 1 Rational cubics are often referred to as singular cubics, because a rational cubic needs to have a singularity. We would like to put emphasis on the fact that rational cubics are regular except in one point and the term singular often indicates degeneracy which is definitely not the case here.

Proof. The equation of the cubic $k_{2}$ is determined by inserting the (homogeneous) trilinear coordinates of

$$
X_{2}=(b c, c a, a b)
$$

(see $[6,7])$ into (2). This yields the corresponding parameter (in the pencil of cubics)

$\delta_{2}=\frac{8 F^{3}}{27 a b c}$

and the equation of the cubic by inserting (3) into (2):

$k_{2}: 27 a b c \xi \eta \zeta-(a \xi+b \eta+c \zeta)^{3}=0$.

Now it is easily verified that $X_{2}$ is the only singular point on $k_{2}$. (Compute the gradient of $k_{2}$ with respect to $(\xi, \eta, \zeta$ ) at $X_{2}$ and recall that a non-degenerate cubic cannot have more than one singularity.) The tangents to $k_{2}$ at $X_{2}$ are given by the equation

$\sum_{\text {cyclic }} a^{2} \xi^{2}-b c \eta \zeta=0$

In order to show that $k_{2}$ is the only singular (nondegenerate) cubic in the pencil (2), the singular points of all cubics in the pencil are computed. For that purpose, first the gradient $\operatorname{grad} k$ is computed. Second, the equation (2) is used to eliminate all variables but one, say $\xi$.
In an intermediate step, the factor $a \xi+b \eta$ is cut out from two resultants. This is admissible, since together with the third resultant $a \xi+b \eta=0$ implies $\xi=0$, and then $\eta=0$ which does not yield a proper point on any of the cubics. In the last elimination step, the final resultant is obtained and reads

$$
27 a^{3} b c \delta\left(27 a b c \delta-8 F^{3}\right)^{2}\left(27 a b c \delta+64 F^{3}\right) \xi^{4}
$$

which can only be zero if either

$$
27 a b c \delta-8 F^{3}=0
$$

or

$$
27 a b c \delta+64 F^{3}=0,
$$

since $a, b, c \neq 0$ (and hence $F \neq 0$, otherwise there is no triangle) and $\xi=0$ only yields the inflection point $I_{2} \in \omega$. The first equation leads precisely to $\delta_{2}$ and $k_{2}$, while the second equation yields

$$
\delta=-\frac{64 F^{3}}{27 a b c}
$$

which determines a regular elliptic cubic. The choice of the variable to be eliminated does not matter.

Among the cubics (2), the cubic $k_{2}$ is the only cubic that can be found on BERNARD GIBERT's page [3], where it is labeled as $\mathcal{L}_{656}$ and has the name Bataille acnodal cubic. Figure 3 shows an example of the Bataille acnodal cubic.

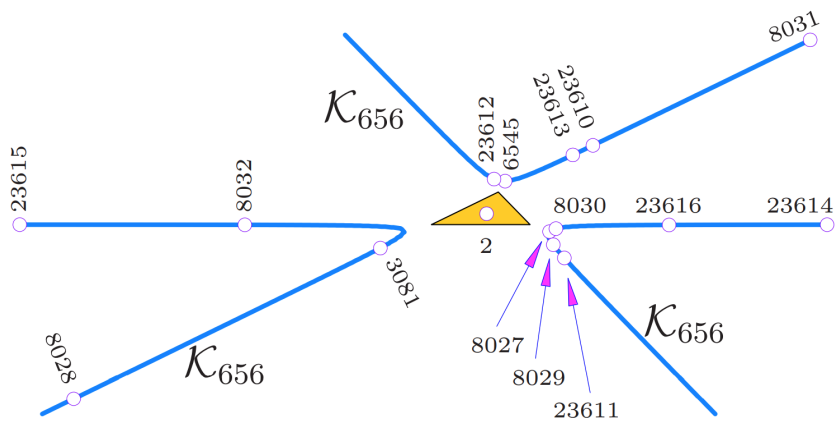

Figure 3: The Bataille acnodal cubic $\mathcal{L}_{656}$ with the 16 known centers on it.

It is easily verified that the Bataille acnodal cubic $\mathcal{K}_{656}$ carries 16 known and labeled triangle centers. These are the centers $X_{i}$ with the Kimberling numbers

$$
\begin{gathered}
2,3081,6545,8027, \ldots, 8032, \\
23610, \ldots, 23616
\end{gathered}
$$

see also [3]). This is by far the highest number of known triangle centers on a distance product cubic.

The cubic $k_{2}=\mathcal{K}_{656}$ admits the surprisingly simple parametrization

$$
\left(b c m^{3},-c a(m+n)^{3}, a b n^{3}\right) \text { with } m: n \neq 0: 0 .
$$


In terms of homogeneous barycentric coordinates, the equation of $k_{2}$ becomes very simple and reads

$$
27 \xi \eta \zeta-(\xi+\eta+\zeta)^{3}=0 .
$$

\subsection{Dual curves, Hessian pencils, and Weierstraß form}

It is well-known that elliptic cubics are of class 6 , while rational cubics are of class 4 or 3 , depending on whether the singularity is a node (isolated or not) or a cusp. The latter case cannot occur: According to Theorem 4, the curve $k_{2}$ is only singular distance product cubic. The equations of the tangents $d_{1}$ and $d_{2}$ at the double point $X_{2}$ are the two (complex conjugate) linear factors of the singular quadratic form (5) and read

$$
\begin{aligned}
& d_{1}: 2 a \xi-b(\sqrt{3} \mathrm{i}+1) \eta+c(\mathrm{i} \sqrt{3}-1) \zeta=0 \\
& d_{2}: 2 a \xi+b(\sqrt{3} \mathrm{i}-1) \eta-c(\mathrm{i} \sqrt{3}+1) \zeta=0 .
\end{aligned}
$$

Only in the case $d_{1}=d_{2}, X_{2}$ becomes a cusp. Since $d_{2}=\overline{d_{1}}$ would imply $d_{1}=\overline{d_{1}}$, and thus, both tangents would have to be real, which is the case only if $b=c=0$. Hence, we have:

Theorem 5 The distance product cubics (2) are of class 6 if $\delta \neq \delta_{2}$. If $\delta=\delta_{2}$, the corresponding distance product cubic is rational and of class 4 .

Especially in the case of $k_{2}=\mathcal{K}_{656}$ there are remarkable connections between the dual $k_{2}^{\star}$ of the distance product cubic $k_{2}$ and some conics deduced from the base triangle $\Delta:$

Theorem 6 The dual curve $k_{2}^{\star}$ of $k_{2}$ is the isogonal image of the Steiner inellipse $e$ and the isotomic image of the triangle's inellipse $i$ with the third Brocard point $X_{76}$ for its Brianchon point (after the canonical identification of homogeneous line and point coordinates).

Proof. The dual curve of $k_{2}$ (or $\mathcal{K}_{656}$ ) has the equation

$k_{2}^{\star}: \sum_{\text {cyclic }}\left(a^{2} u_{1} u_{2}-2 b c u_{0}^{2}\right) u_{1} u_{2}=0$

which can be found by eliminating $\xi, \eta, \zeta$, and $\rho$ from the following system of equations:

$$
\operatorname{grad} k_{2}=\rho \cdot\left(u_{0}, u_{1}, u_{2}\right) .
$$

(Note that $\xi, \eta$, and $\zeta$ are subject to (2) which has to be taken into account during the elimination process.) In order to verify that (6) is the isogonal image of the Steiner inellipse $e$, homogeneous line coordinates $u_{0}: u_{1}: u_{2} \neq$ $0: 0: 0$ are identified with homogeneous point coordinates $\xi: \eta: \zeta \neq 0: 0: 0$. Then, the substitution of $u_{0}=x_{1} x_{2}$ (cyclic, cf. [5]) into (6) indeed yields the equation of the Steiner inellipse

$$
\mathfrak{\imath}\left(k_{2}^{\star}\right)=e: \sum_{\text {cyclic }} a^{2} x_{0}^{2}-2 b c x_{1} x_{2}=0
$$

after canceling the (cyclic symmetric) factor $x_{0}^{2} x_{1}^{2} x_{2}^{2}$ that describes the sides of $\Delta$ 's sides (each with multiplicity 2 ). Finally, it remains to show that the curve $k_{2}^{\star}$ is also the isotomic image of the inellipse with Brianchon point $X_{76}$ (the $3^{\text {rd }}$ Brocard point) and center $X_{141}$ (the complement of the Symmedian point $X_{6}$ ). Applying the isotomic transformation to $k_{2}^{\star}$ means to substitute $u_{0}=b^{2} c^{2} x_{1} x_{2}$ (cyclic, cf. [5]) into (6). In doing so, one finds

$$
\tau\left(k_{2}^{\star}\right)=i: \sum_{\text {cyclic }} a^{6} x_{0}^{2}-2 b^{3} c^{3} x_{1} x_{2}=0 .
$$

Like in the previous cases, the factor $x_{0}^{2} x_{1}^{2} x_{2}^{2}$ is cut out. It is a rather elementary task, to determine the Brianchon point and the center of $i$, see [5].

The curve $k_{2}^{\star}$ has three ordinary cusps at the vertices of $\Delta$ which correspond to the three inflection tangents. Again, homogeneous line coordinates are interpreted as homogeneous point coordinates in the plane of the base triangle and in the underlying projective coordinate system $\left(A, B, C ; X_{1}\right)$.

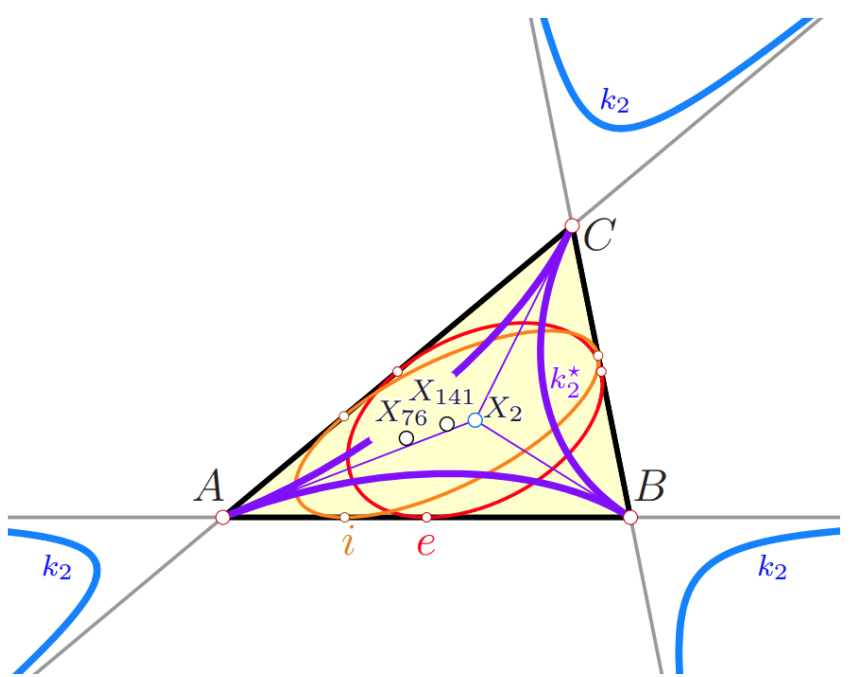

Figure 4: The cubic $k_{2}$, its dual $k_{2}^{\star}$ (interpreted as a point curve), the Steiner inellipse $e$ as the isogonal conjugate $e=\mathrm{l}\left(k_{2}^{\star}\right)$, and the inellipse $i$ as the isotomic conjugate $i=\tau\left(k_{2}^{\star}\right)$.

Figure 4 shows the curves $e, i, k_{2}$, and $k_{2}^{\star}$ mentioned in Theorem 6.

The computation of the dual curves of the non-rational distance product cubics is much more complicated. In contrast to the case of the rational curve $k_{2}$, one cannot rely on a parametrization of the curve. Thus, the homogeneous 
point coordinates $\xi, \eta$, and $\zeta$ have to be eliminated from the following system of equations

$$
\begin{gathered}
\partial_{\xi} k_{\delta}=u_{0} \rho, \partial_{\eta} k_{\delta}=u_{1} \rho, \partial_{\zeta} k_{\delta}=u_{2} \rho, \\
8 F^{3} \xi \eta \zeta-\delta(a \xi+b \eta+c \zeta)^{3}=0 .
\end{gathered}
$$

in order to obtain the implicit equation of the dual curves of (2) (in terms of homogeneous line coordinates $u_{0}: u_{1}: u_{2}$ ). This yields the sextic curves

$$
\begin{gathered}
k_{\delta}^{\star}: 27 \delta^{2} \prod_{\text {cyclic }}\left(b u_{2}-c u_{1}\right)^{2}- \\
-16 \delta F^{3} \prod_{\text {cyclic }}\left(a u_{1} u_{2}+b u_{0} u_{2}-2 c u_{0} u_{1}\right)= \\
=64 F^{6} u_{0}^{2} u_{1}^{2} u_{2}^{2}
\end{gathered}
$$

whose equations depend quadratically on the parameter $\delta \in \mathbb{R} \backslash\{0\}$. The sextic curves (7) have three real and six complex cusps corresponding to the three real and six complex points of inflection on the cubics (2). The nine cusps of (7) form a Hesse configuration $\left(9_{4}, 12_{3}\right)$ if $u_{0}: u_{1}: u_{2}$ are viewed as homogeneous point coordinates.

\subsection{Hessian pencil}

The equation of the Hessian curve $\mathrm{H} c$ of an algebraic curve $c$ with the implicit homogeneous equation $F\left(x_{0}, x_{1}, x_{2}\right)=0$ is given by

$\mathrm{H} c: \operatorname{det}\left(\partial_{i j} F\right)=0$.

It intersects $c$ at ordinary inflection points with multiplicity one (while it intersects $c$ at its singularities with multiplicities larger than 6). Clearly, the Hessian curves of cubics are again cubics.

In the particular case of distance product cubics, one can show:

Theorem 7 The Hessian curves of the distance product cubics (2) form a pencil of cubics which is spanned by the degenerate cubics $e \cup \omega$ (union of the Steiner inellipse $e$ and the line at infinity $\omega)$ and the three side lines of $\Delta$.

Proof. The equations of the Hessian curves of the cubics (2) are computed via (8). This results in

$\mathrm{H} k_{\delta}: \delta \underbrace{\left(\sum_{\text {cyclic }} a \xi\right)}_{\omega} \cdot \underbrace{\left(\sum_{\text {cyclic }} a \xi(2 b \eta-a \xi)\right)}_{\text {Steiner inellipse }}=\frac{8}{3} F^{3} \xi \eta \zeta$.

Since the equations (9) of the Hessian curves are linear in $\delta$, they form a pencil of cubics like the distance product cubics do. The choice of $\delta=0$ yields $\xi \eta \zeta=0$ which is the equation of the three side lines of $\Delta$. Replacing the affine parameter $\delta$ by the homogeneous parameter $\delta_{0}: \delta_{1} \neq 0: 0$ and setting $\delta_{1}=0$ (while $\delta_{0} \neq 0$ ) yields the right-hand side of (9) which factors into the equation of the ideal line $\omega$ and the equation of the Steiner inellipse $e$.

The fact that one factor of the right-hand side of (9) is the equation of $\omega$ clearly shows that for each $\delta \in \mathbb{R} \backslash\{0\}$ the corresponding distance product cubic $k_{\delta}$ and its Hessian curve $\mathrm{H} k_{\delta}$ intersect in the ideal points of $\Delta$ 's side lines (to mention only the real points). This again shows that the three real points of inflection of the distance product cubics (2) are the ideal points of $\Delta$ 's side lines (cf. Theorem 2).

\subsection{Weierstraß form}

The treatment of elliptic cubic curves is usually done in an affine setting. The choice of an affine coordinate frame properly attached to the cubic curve transforms the cubic's equation into the Weierstraß normal form, cf. [10]. Based on this normal form, many computations - especially those related to the group structure on the curve - can be performed in a very simple way.

We set $\zeta=1$ and substitute

$$
\begin{aligned}
\xi & =\frac{2 F^{3}-3 a b c \delta}{6 a^{2} b \delta}-\frac{1}{64 F^{6} a^{2} b \delta}\left(4 F^{3} X+Y\right), \\
\eta & =\frac{2 F^{3}-3 a b c \delta}{6 a b^{2} \delta}-\frac{1}{64 F^{6} a b^{2} \delta}\left(4 F^{3} X-Y\right) .
\end{aligned}
$$

into (2). This yields the Weierstraß normal form of distance product cubics:

$$
\begin{aligned}
k_{W}: & Y^{2}=X^{3}+\frac{2^{8}}{3}\left(3 a b c \delta-F^{3}\right) F^{9} X+ \\
& +\frac{2^{10}}{3^{3}}\left(3^{3}(a b c \delta)^{2}-6^{2} a b c \delta F^{3}+2^{3} F^{6}\right) F^{12} .
\end{aligned}
$$

The $j$-invariant $j(e)$ of an elliptic curve

$$
e: y^{2}=x^{3}+3 p x+2 q
$$

is computed via

$$
j(e)=\frac{2^{6} \cdot 3^{3} \cdot p^{3}}{p^{3}+q^{2}} .
$$

The $j$-invariant of all distance product cubics $k_{\delta}$ equals

$$
j\left(k_{\delta}\right)=\frac{2^{12} F^{3}\left(F^{3}-3 a b c \delta\right)^{3}}{(a b c \delta)^{3}\left(2^{3} F^{3}-3^{3} a b c \delta\right)}
$$

and becomes undetermined if, and only if, $\delta=\delta_{2}$ from (3).

\section{Triangle centers with equal distance product}

The triangle centers listed in KIMBERLING's Encyclopedia of Triangle Centers $[6,7]$ determine cubic curves as loci of points with the equal product of trilinear distances. Finding triangle centers located on the same cubic curve is equivalent to finding triangle centers with the same product of trilinear distances. This would be another classification of triangle centers. 
Surprisingly, among the many known, listed, and in principle arbitrarily numbered triangle centers, there is only a small number of triangle centers that gather on the same cubic.

Until now (as to November 2020), only the following groups of triangle centers located on the same cubic are known:

Theorem 8 The groups of triangle centers with equal distance product to the sides of a triangle are given in Table 1 .

$(1,764),(4,5489),(6,22260),(8,21132)$,
$(2,3081,6545,8027-8032,23610-23616)$,
$(25,394),(42,321,8034),(55,40166),(57,200)$,
$(75,21143),(76,23099),(86,21131),(99,14444)$,
$(145,23764),(324,418),(455,40144),(459,3079)$,
$(649,693),(669,850,32320),(671,14443)$,
$(903,14442),(875,4375,4444),(756,8042)$,
$(1022,3251),(1026,3675),(1422,40212)$,
$(1641,14423),(1647,17780),(1648,5468)$,
$(1649,5466),(1650,4240),(2501,3265)$,
$(3051,8024),(3227,14441),(3233,12079)$,
$(3234,15634),(3239,3676),(3572,27855)$,
$(3733,4036),(4024,7192),(4358,8661)$,
$(4500,4507),(6358,40213),(6384,8026)$,
$(6544,6548),(6557,15519),(8013,8025)$,
$(8023,8039),(14163,14164),(14214,14215)$,
$(14401,34767),(15630,15631),(15632,15635)$,
$(16748,21820),(20696,20700),(21140,23354)$,
$(21438,23655),(27919,40217),(36414,40146)$,
$(40149,40152)$

Table 1: Groups of triangle centers with equal distance product.

Proof. In order to verify the results given in the above theorem, it is sufficient to insert the trilinear representations of the respective centers into to the equations of the cubic curves.

Just inserting trilinear representations of triangle centers into the equations of a particular distance product cubic is not a very efficient search for triangle centers on a cubic. It requires the presence of trilinear representations of triangle centers which is not the case for some triangle centers, cf. [7]. Complicated algebraic expressions involving cube roots or nested square roots can hardly be handled properly with computer algebra systems.

We can improve the search by recalling the following facts: A cubic curve is a triangle cubic if its equations in terms of homogeneous (trilinear or barycentric) coordinates is invariant under the cyclic substitution

$$
a \rightarrow b \rightarrow c \rightarrow a \text { and } \xi \rightarrow \eta \rightarrow \zeta \rightarrow \xi
$$

According to this, the cubics (2) and (9) are triangle cubics. Once a center $C$ on a triangle cubic $k$ is known, one can immediately find a new triangle center $R$ as the intersection of $k$ 's tangent $T_{C} k$ (at $C$ ) with $k$. The point $R$ shall henceforth be called the tangential remainder or simply remainder of $C$. This yields - besides the contact point $C$ with multiplicity 2 - exactly one further point $R$, provided that $C$ is not a point of inflection.

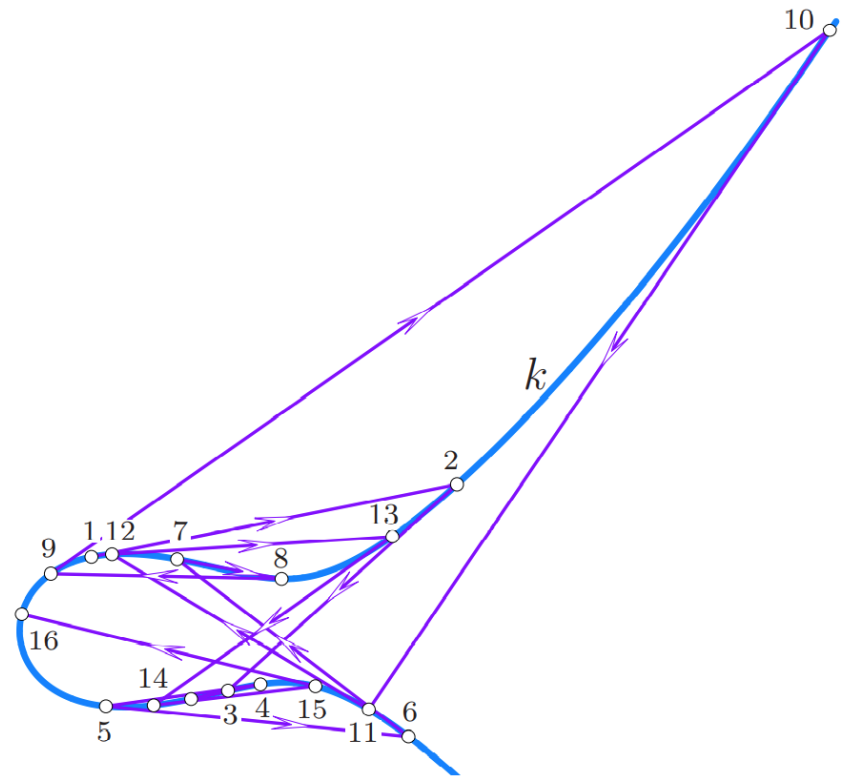

Figure 5: A sequence of (rational) points on an elliptic cubic $k$. Each successor $i+1$ is the intersection $(\neq i)$ of the tangent $T_{i} k$ at $i$ with $k$. Only for points of finite order, such chains are closed.

Once a rational point 1 on the elliptic cubic $k$ is known, a sequence of further rational points can be generated (see Figure 5; note that the chain depicted there is not closed). Only in some rare cases, periodic sequences of remainders (or closed chains) are known and correspond to the groups of finite order on the elliptic curve, see [10].

On the distance product cubics mentioned in Theorem 8, one can observe the following relations between centers and their remainders: 
Type 1: For example, on $k_{1}$, the center $R_{1}=X_{764}$ is the intersection of $k_{1}$ with the tangent $T_{1}$ at $X_{1}$, see Figure 6.

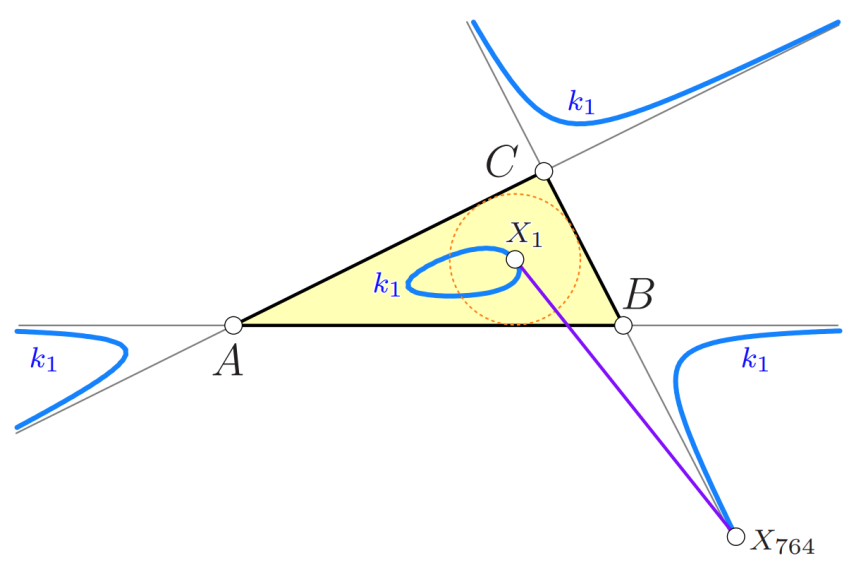

Figure 6: The distance product cubic $k_{1}$ defined by the incenter $X_{1}$. The center $X_{764} \in k_{1}$ is the intersection of $k_{1}$ 's tangent at $X_{1}$.

Note that

$$
\delta_{1}=r^{3},
$$

i.e., the trilinear distance product of the points on the cubic $k_{1}$ equals the cube of $\Delta$ 's inradius $r$. Hence, the triangle center $X_{764}$ is another triangle center with trilinear distance product $\delta_{764}=r^{3}$.

Type 2: The cubic $k_{875}$ is an example of a distance product cubic with three collinear centers on it. These are the centers

$$
X_{875}, X_{4375}, X_{4444},
$$

which are shown together with $k_{875}$ in Figure 7 . In this case the search for further (already known) triangle centers on the cubic fails. None of the tangential remainders is a known triangle center.

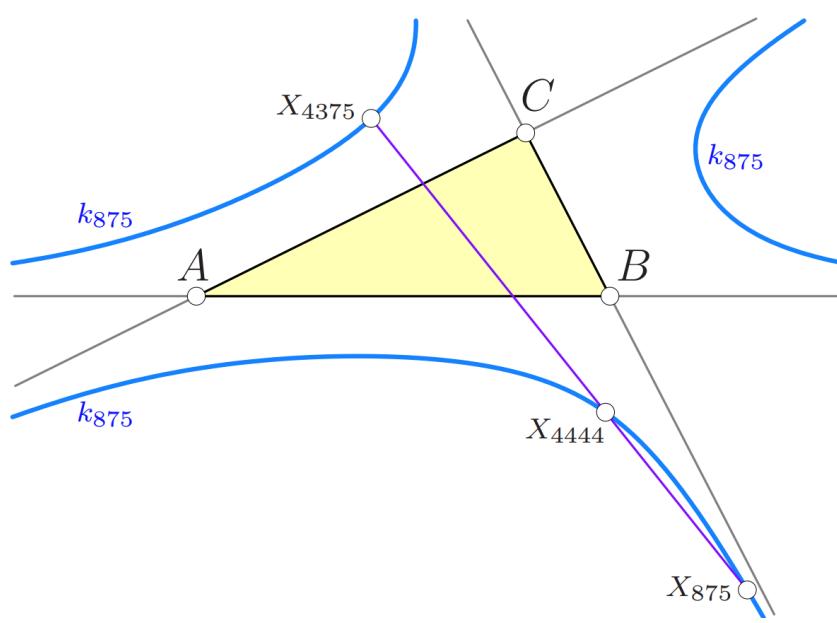

Figure 7: A triple of three collinear centers on $k_{875}: X_{875}$, $X_{4375}$, and $X_{4444}$.

The two centers $X_{649}$ and $X_{693}$ also form the same distance product, and thus, they both lie on the curve $k_{649}$. Clearly, the line

$$
\mathcal{L}_{649,693}:=\left[X_{649}, X_{693}\right]
$$

meets $k_{649}$ in a further center $R$ with trilinear center function

$$
\alpha_{R}=b^{2} c^{2}(b-c)\left(a^{2}-b c\right)^{3} .
$$

Unfortunately, this point cannot be found in KIMBERLING's encyclopedia (cf. [7]) although it has a relatively simple algebraic representation compared to other centers.

Type 3: The cubic $k_{42}$, also hosts three (known) triangle centers. However, the points

$$
X_{42}, X_{321}, X_{8034}
$$

on $k_{42}$ are not collinear. The centers $X_{42}$ and $X_{321}$ have the same remainder

$$
R_{42}=R_{321}=X_{8034},
$$

see Figure 8. Therefore, we could expect to find more triangle centers sending their tangents to $X_{8034}$. Unfortunately, the corresponding polynomial equation of degree 6 has only two rational solutions leading to the already known centers $X_{42}$ and $X_{321}$.

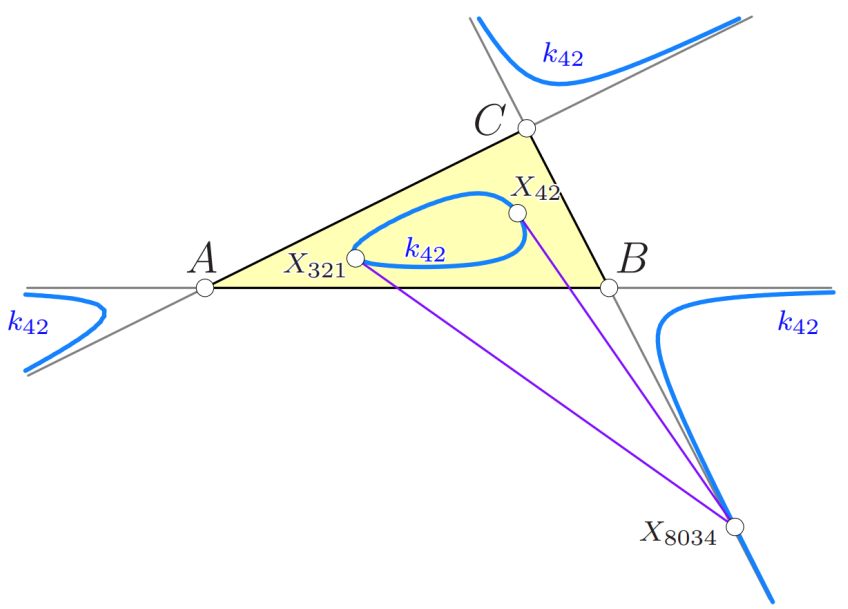

Figure 8: The tangents to $k_{42}$ at $X_{42}$ and $X_{321}$ meet in $X_{8034} \in k_{42}$.

Remark 2 At this point it shall be said that the assignment of numbers (Kimberling numbers) to triangle centers is done rather arbitrarily. Therefore, the configuration of known centers on their particular distance product cubic has no deeper geometric meaning.

Table 2 collects triangle centers and their tangential remainders on their respective distance product cubics. Table 3 gives the trilinear center function of the tangential remainders common to two different (known) triangle centers on their respective distance product cubic. These points do not occur in KIMBERLING's encyclopedia [7]. 


\begin{tabular}{|r|r|r|r|}
\hline$X_{i}$ & $R_{i}$ & $X_{i}$ & $R_{i}$ \\
\hline \hline 1 & 764 & 1641 & 14423 \\
4 & 5489 & 3051,8024 & $R_{3051}$ \\
6 & 22260 & 3227 & 14441 \\
8 & 21132 & 3733,4036 & $R_{3733}$ \\
25,394 & $R_{25}$ & 4240 & 1650 \\
\hline 42,321 & 8034 & 4358 & 8661 \\
55,40166 & $R_{55}$ & 5466 & 1649 \\
57,200 & $R_{57}$ & 5468 & 1648 \\
75 & 21143 & 6358,40213 & $R_{6358}$ \\
76 & 23099 & 6384,8026 & $R_{6384}$ \\
86 & 21131 & 6548 & 6544 \\
\hline 99 & 14444 & 6557,15519 & $R_{6557}$ \\
145 & 23764 & 8013,8025 & $R_{8013}$ \\
324,418 & $R_{324}$ & 8023,8039 & $R_{8023}$ \\
455,40144 & $R_{455}$ & 17780 & 1647 \\
459,3079 & $R_{459}$ & 20696,20700 & $R_{20696}$ \\
671 & 14443 & 16748,21820 & $R_{16748}$ \\
\hline 756,8042 & $R_{756}$ & 23354 & 21140 \\
903 & 14442 & 27919,40217 & $R_{27919}$ \\
1022 & 3251 & 34767 & 14401 \\
1026 & 3675 & 36414,40146 & $R_{36414}$ \\
1422,40212 & $R_{1422}$ & 40149,40152 & $R_{40149}$ \\
\hline & & &
\end{tabular}

Table 2: The tangent of the triangle center $X_{i}$ meets the cubic $k_{i}$ at a further triangle center given in the column $R_{i}$. If this remainder is a known triangle center, then its number is given.

\begin{tabular}{|r|r|}
\hline remainder of & trilinear center function \\
\hline 25,394 & $a\left(b^{2}-c^{2}\right)^{3}\left(a^{2}-b^{2}-c^{2}\right)^{2}$ \\
55,40166 & $(b-c)^{3}(a-b-c)^{2}$ \\
57,200 & $a(b-c)^{3}(a-b-c)\left(a b+c a-b^{2}-c^{2}\right)^{3}$ \\
324,418 & $a^{3}\left(b^{2}-c^{2}\right)^{3}\left(a^{2}-b^{2}-c^{2}\right)^{5}\left(a^{2} b^{2}+a^{2} c^{2}+2 b^{2} c^{2}-b^{4}-c^{4}\right)$ \\
459,3079 & $b c\left(b^{2}-c^{2}\right)^{3}\left(a^{2}-b^{2}-c^{2}\right)^{2}\left(3 a^{4}-2 a^{2}\left(b^{2}+c^{2}\right)-\left(b^{2}-c^{2}\right)\right)^{2}$ \\
\hline 756,8042 & $(b-c)\left(b^{2}-c^{2}\right)^{2}\left(a^{2}-b c\right)^{3}$ \\
3051,8024 & $a^{3}\left(b^{2}+c^{2}\right)\left(b^{2}-c^{2}\right)^{3}$ \\
3733,4036 & $a(b-c)(b+c)^{2}\left(a^{3}(b+c)+a^{2}\left(b^{2}+c^{2}\right)-a\left(b^{3}+c^{3}\right)-b^{4}-c^{4}\right)^{3}$ \\
6358,40213 & $a(b+c)^{2}(b-c)^{3}(a-b-c)^{2}\left(a^{2}-b^{2}+b c-c^{2}\right)^{3}$ \\
6384,8026 & $a(b-c)^{3}(a b+a c-b c)^{2}$ \\
6557,15519 & $b c(b-c)^{3}(a-b-c)(3 a-b-c)^{2}$ \\
8013,8025 & $b c(b-c)\left(b^{2}-c^{2}\right)^{2}(2 a+b+c)$ \\
\hline 8023,8039 & \\
16748,21820 & $a^{7}\left(b^{4}+c^{4}\right)\left(b^{2}-c^{2}\right)\left(b^{2}+c^{2}\right)^{3}$ \\
36414,40166 & $a^{4}(b-c)\left(b^{2}-c^{2}\right)^{2}(a b+a c+2 b c)$ \\
40149,40152 & $a^{3}\left(b^{2}-c^{2}\right)^{3}\left(b^{2}+c^{2}\right)^{3}\left(a^{4}-b^{4}-c^{4}\right)^{2}$ \\
27919,40217 & $a(b+c)(b-c)^{3}(a-b-c)^{2}\left(a^{2}-b^{2}-c^{2}\right)^{2}$ \\
\hline & $b c(b-c)^{3}\left(a b+c a-b^{2}-c^{2}\right)\left(a^{2}-b c\right)^{2}$ \\
\hline
\end{tabular}

Table 3: Tangential remainders common to two centers on distance product cubics. Only those center functions (first trilinear coordinates) of reasonable length are given. 


\section{Tangential remainders for triangle centers}

Table 2 contains a subset of Table 1 and gives a list of triangle centers with their tangential remainders on their distance product cubics. Known centers are given by their Kimberling numbers, while unknown tangential remainders of centers $X_{i}$ are labeled with $R_{i}$.

Table 3 gives the first trilinear center functions of some of the unknown remainders $R_{i}$ mentioned in Table 2, provided that these remainders are common to at least two centers and that the respective center function is of reasonable length.

For the remainders of some of those centers (not appearing in Theorem 8), Table 4 presents the first trilinear center functions together with the respective numeric search value for the triangle

$$
(a, b, c)=(6,9,13)
$$

(in order to simplify the identification and search on [7]).

\section{Outlook, future work, computational problems}

The search of rational points on elliptic triangle cubics, not necessarily distance product cubics, sometimes involves quadratic or cubic field extensions. In the beginning, i.e., for triangle centers with small Kimberling number, most of the triangle centers have trilinear coordinates that are polynomials in $a, b, c$ with integer coefficients. The trilinear representations of the centers $X_{13}, \ldots, X_{18}$ involve $\sqrt{3}$ which does not cause problems in symbolic computations.

Square roots show up in the trilinear representations that involve half-angle functions. In order to handle expressions that involve the area function

$$
F=\frac{1}{4} \sqrt{(a+b+c)(b+c-a)(c+a-b)(a+b-c)}
$$

of the base triangle, we add $F$ as a further element of the coefficient ring. We have to add the square roots of 3 and 5 to the ring of coefficients if multiples of angles of $\frac{\pi}{3}$ and $\frac{\pi}{5}$ are ingredients of the construction of some center: $F$ as well as $\sqrt{3}$ appear in the trilinear representations of the centers

$$
X_{13}, \ldots, X_{18}
$$

$\left(1^{\text {st }}\right.$ and $2^{\text {nd }}$ isogonic center, $1^{\text {st }}$ and $2^{\text {nd }}$ isodynamic point, $1^{\text {st }}$ and $2^{\text {nd }}$ Napoleon point). The trilinear representations of $X_{1139}$ and $X_{1140}$ (Outer and Inner Pentagon point) involve $\sqrt{5}$.

The triangle centers

$$
X_{173}, X_{174}, X_{258}, X_{351}, \ldots, X_{364}
$$

(related with isoscelizers points), involve square roots of $a, b, c$ and sine and cosine of half angles. The trilinear representations of the Square Root point and its isogonal conjugate

$$
X_{365} \text { and } \mathrm{X}_{366}
$$

involve even $\sqrt{a}, \sqrt{b}$, and $\sqrt{c}$.

Triangle centers whose trilinear coordinate functions involve cube roots are also not tested whether or not they share their trilinear distance prodcut with others: These are centers like

$$
X_{356}, X_{357}, X_{358}
$$

(Morley point, $1^{\text {st }}$ and $2^{\text {nd }}$ Morley-Taylor-Marr center), and the Burgess point

$$
X_{1133}, \quad \alpha_{1133}=\sin \frac{\pi-A}{3} \operatorname{cosec} \frac{\pi+A}{3} .
$$

Here and in the following, the letter $A$ denotes the measure of the interior angle at the vertex $A$. Thirds of angles are equivalent to roots of cubic polynomials, and thus, to field extensions of degree 3 .

There are triangle centers that could be termed transcendental, for example:

$$
X_{359} \text { and } X_{360} \text {, }
$$

i.e., Hofstaedter One point and the Hofsteadter Zero point with the trilinear center functions

$$
\alpha_{359}=\frac{a}{A} \text { and } \alpha_{360}=\frac{\mathrm{A}}{\mathrm{a}} .
$$

Their trilinear distance product are not compared with that of other centers, since they will hardly produce the same product as a polynomial center will do. This is also true for the Pure Angles center, the isogonal conjugate of the Point Algenib, the point Algenib, and the Exterior Angle Curvature Centroid, i.e., for the centers

$$
X_{1049}, X_{1085}, X_{1028}, \text { and, } X_{1115}
$$

with the respective trilinear triangle center functions

$$
\begin{gathered}
\alpha_{1049}=A, \quad \alpha_{1085}=A^{2}, \\
\alpha_{1028}=A^{-2}, \quad \alpha_{1115}=\frac{\pi-A}{a} .
\end{gathered}
$$

The trilinear coordinates of triangle centers $X_{40297}, \ldots$, $X_{40305}$ which are related to the power curve involve even logarithms, and thus, their trilinear distance products will not be equal to that of algebraic centers. Besides, $X_{40297}$, $X_{40298}, X_{40299}$ are points at infinity.

Future work is guaranteed, since the ETC is growing continuously. Every day a few new triangle centers are added, awaiting to be tested whether or not they share their trilinear distance product with other centers. 


\begin{tabular}{|c|c|c|c|c|c|}
\hline \multicolumn{6}{|c|}{ Tangential remainders } \\
\hline$i$ & trilinear center function & search-6-9-13 value & $i$ & trilinear center function & search-6-9-13 value \\
\hline 3 & $a\left(b^{2}-c^{2}\right)^{3}\left(a^{2}-b^{2}-c^{2}\right)^{4}$ & 62.32822092189367 & 44 & $\begin{array}{r}(b-c)^{3}(2 a-b-c) \cdot \\
\cdot(a-2 b-2 c)^{3}\end{array}$ & 14.27293379474638 \\
\hline 5 & $\begin{array}{r}b c\left(a^{2}-b^{2}-c^{2}\right)^{3} . \\
\cdot\left(a^{2}\left(b^{2}+c^{2}\right)-\left(b^{2}-c^{2}\right)^{2}\right)\end{array}$ & 5.08075321118240 & 45 & $\begin{array}{r}(b-c)^{3}(a-2 b-2 c) \cdot \\
\cdot(2 a-b-c)^{3}\end{array}$ & 17.54515704225141 \\
\hline 7 & $b c(a-b-c)^{2}(b-c)^{3}$ & -7.25734135716562 & 55 & $\begin{array}{l}a(b-c)^{3}(a-b-c) \cdot \\
\cdot\left(a b+a c-b^{2}-c^{2}\right)^{3}\end{array}$ & 10.54263482016639 \\
\hline 9 & $(b-c)^{3}(a-b-c)^{4}$ & -34.83523851439501 & 56 & $\begin{array}{l}a(b-c)^{3}(a-b-c)^{2} . \\
\left(a b+a c+b^{2}+c^{2}\right)^{3}\end{array}$ & -2.26993220290925 \\
\hline 10 & $b c\left(b^{2}-c^{2}\right)(b-c)^{2}$ & -2.36106585447966 & 57 & $(b-c)^{3}(a-b-c)^{2}$ & -3.71034493822937 \\
\hline 11 & $\begin{array}{r}b c(a-b-c)(b-c)^{5} . \\
\quad\left(a b+a c-b^{2}-c^{2}\right)^{3}\end{array}$ & 10.57329984291265 & 58 & $\begin{array}{r}a(b-c)\left(b^{2}-c^{2}\right)^{2} . \\
\left(a b+a c+b^{2}+b c+c^{2}\right)^{3}\end{array}$ & -1.45795477222064 \\
\hline 20 & $\begin{array}{r}b c\left(3 a^{4}-2 a^{2}\left(b^{2}-c^{2}\right)-\right. \\
\left.-\left(b^{2}-c^{2}\right)^{2}\right)\end{array}$ & 176.82315634331623 & 63 & $\begin{array}{r}(b-c)^{3}\left(a^{2}-b^{2}-c^{2}\right) \cdot \\
\cdot(a-b-c)^{3}\end{array}$ & -57.29426844986488 \\
\hline 21 & $\begin{array}{r}(b-c)(a-b-c) \cdot \\
\cdot\left(b^{2}-c^{2}\right)^{2}\left(a^{2}-b^{2}-c^{2}\right)^{3}\end{array}$ & 29.19736986425203 & 65 & $\begin{array}{r}(b+c)(b-c)^{3}(a-b-c)^{2} \\
\quad \cdot\left(a^{2}+a b+a c+2 b c\right)^{3}\end{array}$ & -4.33523005169485 \\
\hline 22 & $\begin{array}{r}a\left(b^{2}-c^{2}\right)^{3}\left(a^{4}-b^{4}-c^{4}\right) \\
\cdot\left(a^{2}-b^{2}-c^{2}\right)^{3}\end{array}$ & -223.89401674331905 & 66 & $b c\left(b^{4}-c^{4}\right)^{3}\left(a^{4}-b^{4}-c^{4}\right)^{2}$ & 77.10999885446101 \\
\hline 23 & $\begin{array}{r}a\left(b^{2}-c^{2}\right)^{3}\left(a^{2}-b^{2}-c^{2}\right)^{3} \\
\cdot\left(a^{4}-b^{4}+b^{2} c^{2}-c^{4}\right)\end{array}$ & -28.06151358971226 & 75 & $a(b-c)^{3}$ & -0.86891361128715 \\
\hline 24 & $\begin{array}{r}a\left(b^{2}-c^{2}\right)^{3}\left(a^{2}-b^{2}-c^{2}\right)^{2} \\
\cdot\left(a^{4}-2 a^{2}\left(b^{2}+c^{2}\right)+b^{4}+c^{4}\right)\end{array}$ & 14.62241731719719 & 76 & $a^{3}\left(b^{2}-c^{2}\right)^{3}$ & -0.5829933417003 \\
\hline $\begin{array}{r}25, \\
394\end{array}$ & $a\left(b^{2}-c^{2}\right)^{3}\left(a^{2}-b^{2}-c^{2}\right)^{2}$ & -5.02132305357783 & 81 & $(b+c)^{2}(b-c)^{3}$ & -1.7256277235825 \\
\hline 27 & $\begin{array}{l}b c(b+c)^{2}(b-c)^{3} \cdot \\
\quad\left(a^{2}-b^{2}-c^{2}\right)^{2}\end{array}$ & -23.86418264611576 & 82 & $\begin{array}{r}(b-c)^{3}\left(b^{2}+c^{2}\right)^{2} \cdot \\
\left(a^{2}+b^{2}+b c+c^{2}\right)^{3}\end{array}$ & -2.7552998535293 \\
\hline 28 & $(b+c)^{2}(b-c)^{3}\left(a^{2}-b^{2}-c^{2}\right)^{2}$ & -7.98316487555603 & 83 & $b c\left(b^{2}+c^{2}\right)^{2}\left(b^{2}-c^{2}\right)^{3}$ & -6.1052017302889 \\
\hline 31 & $a^{2}(b-c)^{3}\left(b^{2}+b c+c^{2}\right)^{3}$ & -1.14992786985712 & 85 & $a(b-c)^{3}(a-b-c)^{5}$ & -52.6095855034850 \\
\hline 32 & $a^{3}\left(b^{2}-c^{2}\right)^{3}\left(b^{2}+c^{2}\right)^{3}$ & -0.94646193660754 & 86 & $b c(b+c)^{2}(b-c)^{3}$ & -2.8159081633291 \\
\hline 37 & $a^{3}(b+c)(b-c)^{3}$ & -0.49015639611069 & 87 & $(b-c)^{3}(a b+a c-b c)^{5}$ & -0.0012017084959 \\
\hline 38 & $\left(b^{2}+c^{2}\right)(b-c)^{3}\left(a^{2}-b c\right)^{3}$ & -35.23250675370688 & 88 & $(b-c)^{3}(2 a-b-c)^{2}$ & 3.8941592730472 \\
\hline 39 & $a^{7}\left(b^{2}+c^{2}\right)\left(b^{2}-c^{2}\right)^{3}$ & -0.19164804132159 & 89 & $(b-c)^{3}(a-2 b-2 c)^{2}$ & -2.2543301181918 \\
\hline 43 & $(b-c)^{3}(a b+a c-b c)$ & -0.19597779819792 & 94 & $\begin{array}{r}a^{3}\left(b^{2}-c^{2}\right)^{3} \\
\cdot\left(a^{2}-b^{2}-b c-c^{2}\right)^{2}\end{array}$ & -17.011141733428 \\
\hline 98 & $\begin{array}{r}b c\left(b^{2}-c^{2}\right)^{3} \\
\cdot\left(a^{2} b^{2}+a^{2} c^{2}-b^{4}-c^{4}\right)^{2}\end{array}$ & 56.616091423347 & 99 & $b c\left(b^{2}-c^{2}\right)^{2}\left(2 a^{2}-b^{2}-c^{2}\right)^{3}$ & 14.834689935205 \\
\hline 100 & $(b-c)^{2}\left(a b+a c-b^{2}-c^{2}\right)^{3}$ & 10.408092032867 & 105 & $(b-c)^{3}\left(a b+a c-b^{2}-c^{2}\right)^{2}$ & 10.776490698571 \\
\hline
\end{tabular}

Table 4: Tangential remainders (not listed in KIMBERLING's encyclopedia of some triangle centers. The remainders of centers are added to this list only if their trilinear center function is of reasonable length.

\section{References}

[1] W. Burau, Algebraische Kurven und Flächen. IAlgebraische Kurven der Ebene, De Gruyter, Berlin, 1962.

[2] B. Gibert, Cubics in the Triangle Plane, https://bernard-gibert.pagesperso-orange.fr/

[3] B. GIBERT, Bataille acnodal cubic, https://bernard-gibert.pagesperso-orange.fr/ Exemples/k656.html
[4] B. GIBERT, Thomson cubic, https://bernard-gibert.pagesperso-orange.fr/ Exemples/k002.html

[5] G. Glaeser, H. Stachel, B. Odehnal, The Universe of Concis - From the ancient Greeks to $21^{\text {st }}$ century developments, Springer-Spektrum, SpringerVerlag, Heidelberg, 2016.

[6] C. Kimberling, Triangle Centers and Central Triangles, (Congressus Numerantium Vol. 129) Utilitas Mathematica Publishing, Winnipeg, 1998. 
[7] C. Kimberling, Encyclopedia of Tr angle Centers, http://faculty.evansville.edu/ck6/ encyclopedia

[8] B. OdehnAL, Generalized Gergonne and Nagel Points. Beitr, Alg. Geom. 51/2 (2010), 477-491.

[9] B. Odehnal, A one-parameter family of triangle cubics, to appear in: Proc. ICGG 2020.

[10] J.H. Silverman, J.T. TATE, Rational Points on Elliptic Curves, Springer, New York, 2015.

\section{Boris Odehnal}

orcid.org/0000-0002-7265-5132

e-mail: boris.odehnal@uni-ak.ac.at

University of Applied Arts Vienna

Oskar-Kokoschka-Platz 2, A-1100 Vienna, Austria 